\title{
STUDYING THE MAGNETISM OF THE QUIET SUN: DIAGNOSTIC TECHNIQUES AND RESULTS
}

\author{
E. Khomenko ${ }^{1,2}$, R. Kostik ${ }^{2}$ \\ ${ }^{1}$ Instituto de Astrofísica de Canarias, E-38200, La Laguna, Tenerife, Spain \\ ${ }^{2}$ Main Astronomical Observatory, NASU, 27 Zabolotnogo Str., Kyiv, UA-03680, Ukraine
}

(Received September 14, 2006; in final form - December 1, 2006)

\begin{abstract}
Most of our knowledge of solar surface magnetism comes from the analysis of polarization spectra. The Stokes spectra contain detailed information on the structure and dynamics of the magnetized photospheric plasma and its interaction with convection, i. e., magnetoconvection. The interpretation of high-resolution observations requires sophisticated techniques such as radiative transfer of polarized light in 3D model atmospheres. On the other hand, 3D magnetoconvection simulations include elaborated physics and are becoming sufficiently realistic to make predictions about the complex processes that take place in the Sun's magnetized atmosphere. This paper concentrates on the diagnostics of the magnetic fields in quiet solar photospheric regions outside sunspots and active regions. Until recently the influence of the magnetic field on the dynamics of these regions was considered unimportant. However, it turns out that a considerable amount of magnetic energy is probably stored in the "quiet" Sun. The issue of quiet solar magnetism remains open and is much debated in the literature.
\end{abstract}

Key words: Sun, photosphere, magnetic field, polarization.

PACS number(s): 96.60.-j, 96.60.Mz, 96.60.Hv

\section{STOKES DIAGNOSTICS}

One of the most powerful tools for the diagnostics of magnetic fields in the Sun is the interpretation of spectropolarimetric observations (Landi Degl'Innocenti \& Landolfi (2004)). Magnetic fields affect the polarization state of solar radiation via the Zeeman and Hanle effects.

The use of the Zeeman effect became a standard technique for the magnetic field measurements during the last century (see, for example, the review by Solanki (1993)). It has an advantage that a mere detection of polarization implies the presence of a magnetic field.

Due to the relative simplicity of the physics of the Zeeman effect, many approximate methods have been developed in order to derive information about the magnetic field under the assumption that the influence of other atmospheric parameters on spectral lines is less important. An example is the magnetic line ratio technique, which was applied first by Stenflo (1973) to the Fe I $\lambda 5247, \lambda 5250 \AA$ line pair in order to measure the field strength of spatially unresolved magnetic elements in the network.

Zeeman-based measurements have also disadvantages. Firstly, even at high spatial resolution not all the fields are resolved and the resulting polarization signal is affected by the cancellations produced by magnetic elements with opposite polarities in the resolution element. Secondly, the polarization signal is smaller for spectral lines with a larger Doppler width. For most photospheric lines this implies that, typically, fields stronger than 200-300 G can be reliably measured. It also makes it insufficient to determine the magnetic field vector in the solar corona.
Another effect that produces linear polarization in spectral lines is anisotropic radiation pumping. If the radiation illuminating an atom is anisotropic it can produce population imbalances among the magnetic $M_{J}$ sublevels of the $J$ level. Since the populations of the magnetic sublevels are different, the contributions from $\sigma$ and $\pi$ transitions do not cancel out and polarization is observed without the need for a magnetic field.

The presence of a magnetic field alters the polarization state of the radiation via the Hanle effect. The most recent reviews on the Hanle effect and its applications can be found in Trujillo Bueno (2001, 2003a,b); Trujillo Bueno \& Manso Sainz (2002). Briefly, in the 90degree scattering case observed at the solar limb, the magnetic field inclined with respect to the direction of the symmetry axis of the radiation produces a decrease of the linear polarization amplitude and a rotation of the polarization direction. At the solar disk center, no scattering polarization is created in the absence of the magnetic field. However, an inclined magnetic field breaks the symmetry of the scattering problem and can produce linear polarization via the Hanle effect.

The Hanle effect produces a significant contribution when the Zeeman splitting is of the order of the natural width of the spectral line. Therefore, the Hanle effect is sensitive to the fields in the range from $10^{-3}$ up to about $300 \mathrm{G}$, depending on the transition.

An advantage of the Hanle effect is that it is sensitive to the presence of complex magnetic fields with mixed polarities at sub-telescopic scales. It is sensitive to much weaker magnetic fields than the Zeeman effect, independently of the spectral line width. However, a disadvantage of Hanle-based measurements is that they cannot distinguish between magnetic strengths larger than the 
saturation limit (e.g., $200 \mathrm{G}$ for the Sr I $4607 \AA$ line). Another disadvantage is that the quantum theory of polarization needs to be applied, which is complicated and makes the interpretation less straightforward than in the case of the Zeeman effect.

Nevertheless, in recent years the Hanle effect has changed from being considered simply an exotic theoretical (de)polarization mechanism to a powerful tool for the diagnostics of solar magnetism. The applications of the Hanle effect are efficient in the regions of weak fields or in hot chromospheric and coronal plasmas, where the Zeeman effect does not provide enough sensitivity.

\section{RETRIEVING INFORMATION FROM STOKES SPECTRA}

\section{A. Stokes inversion}

The inversion of Stokes profiles has proved to be a robust technique for the analysis of spectro- polarimetric observations based on the Zeeman effect (see, for example, the reviews by del Toro Iniesta \& Ruiz Cobo (del Toro Iniesta \& Ruiz Cobo, 1996) and Socas-Navarro (2001)). Generally, the relation between the observables (Stokes parameters) and the atmospheric quantities is non-linear and cannot be expressed analytically. To be able to solve an inversion problem, the radiative transfer equation (RTE) is linearized assuming small variations of the atmospheric parameters. The linearization allows to express the variations of the outcoming Stokes spectra in terms of response functions (RFs; Ruiz Cobo \& del Toro Iniesta, 1992, 1994). RFs give information about the height and wavelength location of the response of the Stokes spectra to the perturbations in the parameter $x$. Based on this information, an inversion code performs the minimization of a merit function, i. e., the difference between the observed and synthetic Stokes spectra. The model atmosphere, or the set of free parameters of the inversion, is modified iteratively and finally converges to the model that reproduces an observed profile to some degree of accuracy.

The inversion strategies described in the literature can be classified depending on their degree of sophistication. The simplest inversion is based on the MilneEddington (ME) approximation (Skumanich \& Lites (1987)), which assumes a linear variation with optical depth of the source function and a constancy of all the other atmospheric parameters, which allows for the analytical solution of the Unno-Rachkovsky equations (Landi Degl'Innocenti (1992); Rachkovsky (1967); Unno (1956)). Since there are no gradients with height, the asymmetry of the Stokes profiles cannot be fitted. The ME inversion has the advantage of simplicity, speed and a small number of free parameters. However, the results of the ME inversion are reliable only for the magnetic and velocity fields of a simple structure. The results on thermodynamics can be affected by a tradeoff between the magnetic and line formation parameters (Westendorp Plaza et al. (1998)).

Based on ME atmospheres, fast inversion techniques have been developed (Socas-Navarro et al. (2001); Socas-
Navarro (2005b)). These are extremely useful for the routine processing of huge amounts of data obtained from space missions or daily monitoring observations from ground-based telescopes. As for today, two alternative methods of fast inversion have been applied: artificial neural networks and principle component analysis (PCA; Rees et al. (2000); Socas-Navarro et al. (2001); SocasNavarro (2005b)). Both techniques need a database of precomputed profiles and their corresponding models that represents, in a statistical way, all possible observed profiles. In order to limit the number of free parameters, these profiles are computed using ME atmospheres. The best correspondence between the observed and precomputed profiles is then looked for.

Considerably more realism is reached in the SIR inversion code, which allows for gradients of the physical magnitudes along the line of sight (Ruiz Cobo \& del Toro Iniesta (1992)). A similar strategy was also applied in the SPINOR code developed by Frutiger et al. (1999, 2000). This inversion is based on the information provided by the response functions. The free parameters $T, P_{e}, V_{\mathrm{LOS}}, V_{\mathrm{mic}}, V_{\mathrm{mac}}, \mathbf{B}$ are calculated at several points (called nodes) in optical depth. Thus, the number of free parameters is significantly larger than in the case of the ME inversion. The atmosphere is assumed to be in vertical hydrostatic (or MHS) equilibrium. Several, magnetized and non-magnetized, components with different thermodynamics are permitted to co-exist in a resolution element producing an observed Stokes profile. The limitation of the SIR and SPINOR inversions is the assumption of local thermodynamical equilibrium (LTE), which makes the solution of the RTE easier, but is at the same time inappropriate for the strong lines formed high in the atmosphere (or those sensitive to non-LTE effects).

The MIcroStructured Magnetic Atmosphere (MISMA) inversion is an alternative way of treating spatially unresolved magnetic structures (Sánchez Almeida (1997); Sánchez Almeida \& Lites (2000)). The MISMA atmosphere is representative of a conglomerate of thin $(\ll 100$ $\mathrm{km}$ ) vertical flux tubes having the same temperature as the surrounding atmosphere at all heights. There is a lateral pressure balance between the different magnetic and non-magnetic components, and hydrostatic equilibrium is assumed along the magnetic field lines. This constraint provides the vertical stratification of the magnetic field.

The shortcoming of the assumption of the LTE is overcome by the non-LTE inversion code developed by SocasNavarro et al. $(1998,2000)$. The method used for the inversion is similar to SIR (Ruiz Cobo \& del Toro Iniesta, 1992), but the assumption of LTE is relaxed in the solution of the RT equation. The non-LTE inversion methods are extremely important since they can be applied to recover the thermal and magnetic structure of the solar chromosphere. A recent application of the non-LTE inversion to the Ca II and Fe I lines at $850 \mathrm{~nm}$ has allowed Socas-Navarro (2005a) to infer the thermal structure and vector current densities in the photosphere and chromosphere of a sunspot. 


\section{B. Hanle effect diagnostics}

In the case of the Hanle effect, the magnetic field can be inferred based on a single- or multi-line approach. If only one spectral line is available, one has to compare the observed linear polarization amplitude with the amplitude corresponding to the zero-field reference case, obtained from the radiative transfer modeling under nonLTE conditions (see, for example, Bommier et al. (2005); Faurobert et al. (2001, 1995); Trujillo Bueno et al. (2002, $2004,2005)$ ). The difficulty in this case is that the solution depends on the model atmosphere and other free parameters, such as microturbulent and macroturbulent velocities, collisional rates, etc., thus introducing a bias into the magnetic field measurements. The way to reduce the number of free parameters is to perform $3 \mathrm{D}$ radiative transfer calculations in realistic model atmospheres from numerical simulations (Trujillo Bueno et al. (2004)).

The multi-line case is based on line ratio techniques for the Hanle effect. A careful selection of the spectral lines must be done in order to guarantee the same sensitivity to all the atmospheric parameters except for the magnetic field (see, for example, Manso Sainz et al. (2005)).
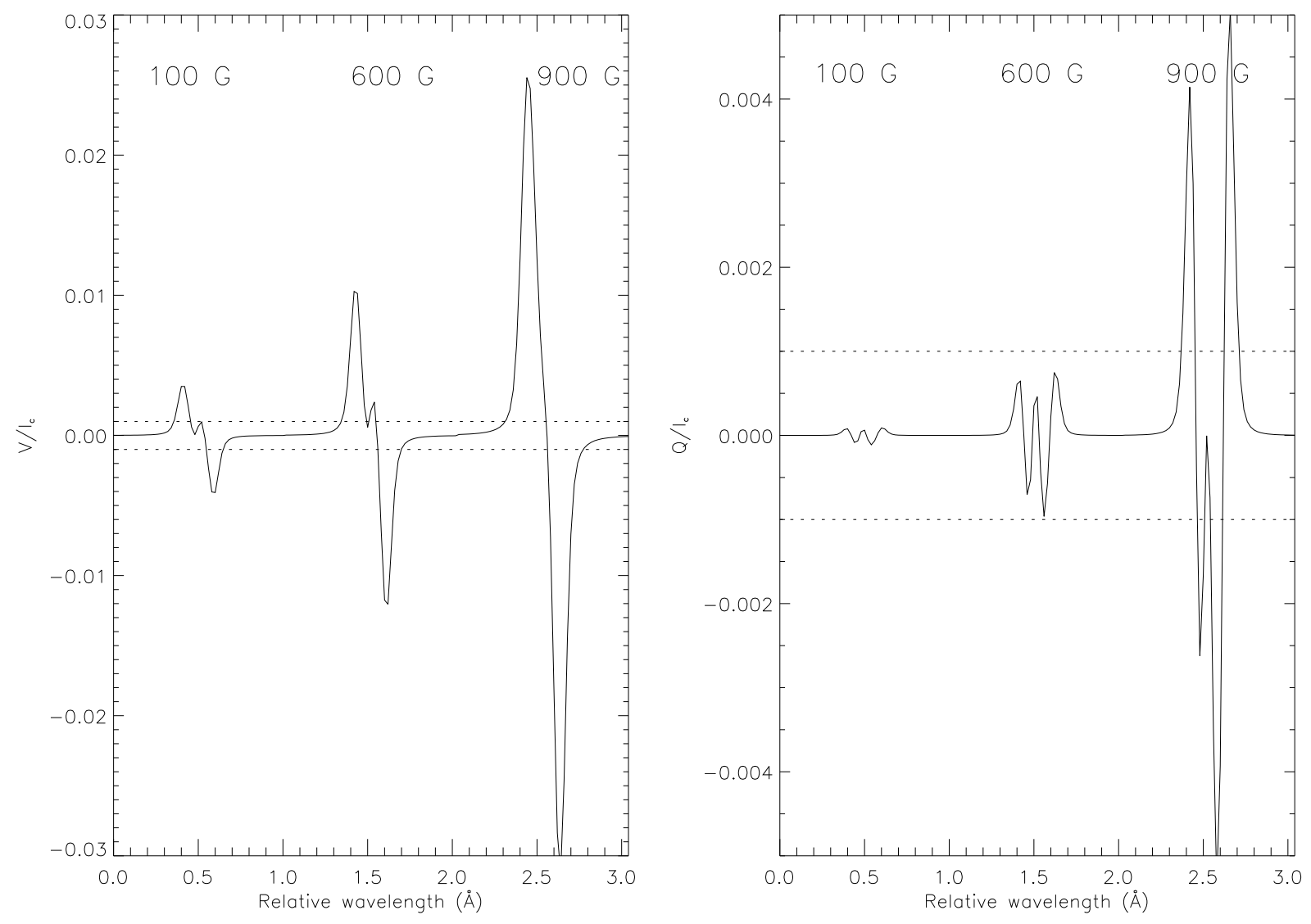

Fig. 1. Stokes $V$ (left) and $Q$ (right) profiles for the Mn I $5537.7 \AA$ line. Three cases of the same spectral line are displaced horizontally by $1 \AA$ for clarity purposes. They are computed using ME atmospheres with constant magnetic field strengths of, from left to right, 100, 600, and 900 G. From López Ariste et al. (2002).

\section{Hyperfine structure}

An alternative possibility to extract information about the magnetic field strength in the photosphere from Stokes spectra was suggested by López Ariste et al. (2002). It is based on the Zeeman effect of the hyperfine structure (HFS) of particular atoms. Due to the interaction between the total angular momentum $J$ and a nuclear angular momentum $I$ for some atoms, the $J$ atomic level is split into $F$ sub-levels in the absence of a magnetic field. When a magnetic field is present, each $F$ level is further split into $M$ magnetic sub-levels. As a result, the Stokes profile has a complicated structure and multiple peaks. An example of this structure is given in Figure 1. The Stokes $V$ shows an inversion in the line core and multiple peaks appear in Stokes $Q$ and $U$. An increase of the field leads to a crossing between the $M_{F}$ sub-levels of each $F$ level and the relative amplitude of the HFS components changes. For the Mn I line shown in Fig. 1, the multiple peaks in Stokes $V$ disappear at 900 G. This suggests the possibility of estimating the strength of the magnetic field directly from the shape of the Stokes profiles.

Note that the presence of HFS depends on the intrinsic 
field strength, not on the magnetic filling factor or flux. The ratio of the peaks of the HFS gives a direct measure of the field strength similar to the line ratio method applied to different lines (Stenflo, 1973), but with the advantage of not being affected by their different sensitivity to the other atmospheric parameters (López Ariste et al. $(2002,2006))$. The disadvantage is that the additional peaks have low amplitudes that can be confused with a noise.

\section{MHD simulations}

Another approach for studying magnetic fields in the Sun is based on the numerical modeling of magnetoconvection (see the reviews by Schüssler (2001, 2003), and references therein). Realistic magneto-convection simulations involve the solution of the full compressible MHD equations including elaborated physics, such as multidimensional radiative transfer or partial ionization and, thus, can make clear predictions about the complex processes that take place in the Sun's magnetized atmosphere. The results of simulations of the solar photosphere and the uppermost layers of the convection zone can be directly compared with observations (Khomenko et al. (2005b); Sánchez Almeida et al. (2003a); Sheminova (2004); Stein \& Nordlund (2003)). Existing simulations of the photospheric magnetic structure in 2 and 3 dimensions show similar properties as the observed solar magnetoconvection: flux expulsion and field intensification in intergranular lanes, spatial scales of polarity changes, network bright points and limb faculae regions, fine structures in intergranules, etc. (Carlsson et al. (2004); Emonet \& Cattaneo (2001); Gadun et al. (2001); Grossmann-Doerth et al. (1998); Keller et al. (2004); Stein \& Nordlund (2003); Vögler et al. (2005)).
In recent years successful attempts have been made to extend realistic MHD simulations to the chromospheric layers (Schaffenberger et al. (2006); Wedemeyer-Böhm et al. (2006)). Chromospheric simulations show very different dynamical scales of the plasma below and above the $\beta=1$ level. Above this level, the atmosphere is much more dynamic and shock formation occurs frequently.

Simulations can be compared to observations by means of spectral synthesis. An advantage of the simulated spectra is that we know exactly the atmosphere producing this or other type of profile. The disadvantage of the simulations is the same as their advantage, i. e., their realism and complexity. It is often difficult to separate the different physical processes primarily responsible for an observed event. The synthetic spectra must be reduced and studied statistically, similarly to observations.

\section{NETWORK vs INTER-NETWORK FIELDS}

The magnetized solar plasma is organized on different temporal and spatial scales, from super- granulation down to the fine structures seen in high-resolution magnetograms in intergranular lanes (Berger et al. (2004)). The average unsigned flux decreases with decreasing scale of convection. It is largest at the supergranular borders, intermediate at the borders of mesogranules (see, for example, Domínguez Cerdeña et al. (2003)) and stays almost at the detection limit for the granular magnetic fields (Lin \& Rimmele (1999)). There is almost an order of magnitude difference between the flux in the network and inter-network areas. Bright points observed in intergranular lanes in the $G$-band follow the strong magnetic field concentrations located mostly at the supergranular borders or in plage areas (Carlsson et al. (2004); Keller et al. (2004); Schüssler et al. (2003)).
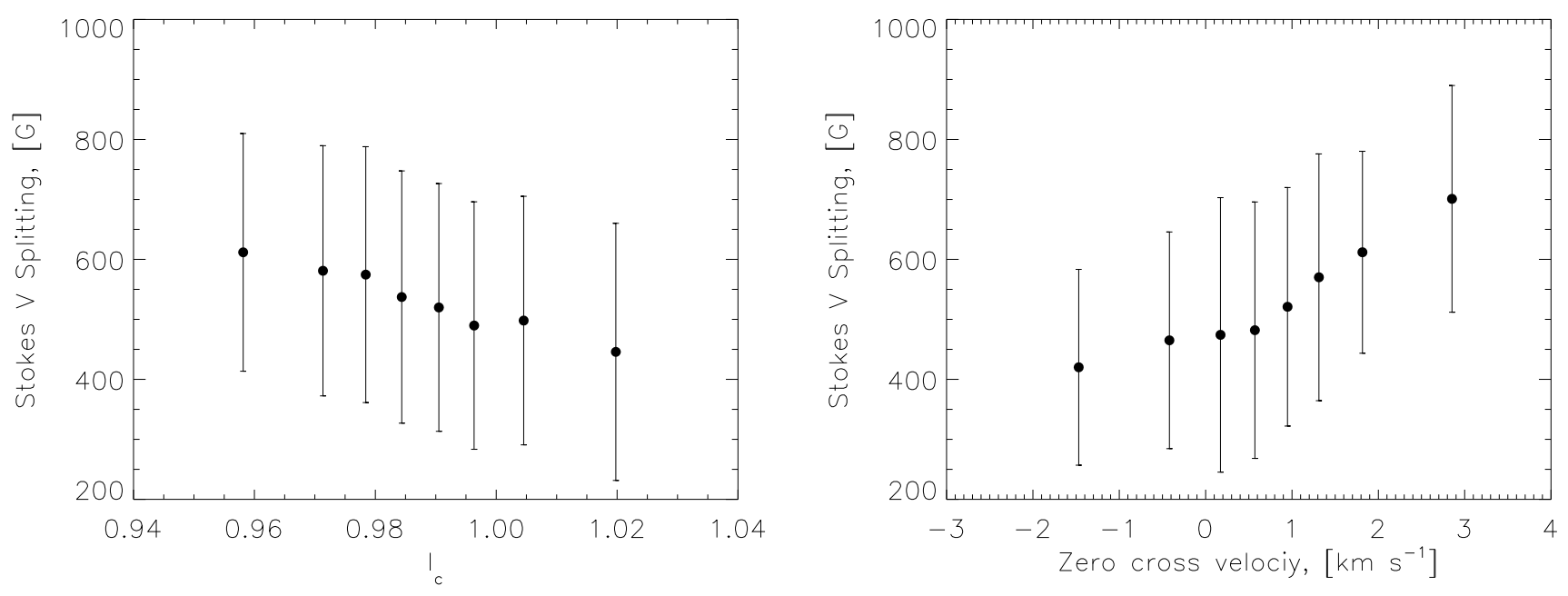

Fig. 2. Stokes $V$ splitting as a function of continuum intensity (left) and Stokes $V$ zero-crossing velocity (right) from the inter-network observations in the infrared Fe I $1.56 \mu \mathrm{m}$ lines (Khomenko et al. (2003)). Error bars show standard deviation within each interval. 
There has been much confusion in the literature regarding the definition of "quiet Sun". By "quiet Sun" one understands both network and internetwork areas, whose flux level and, possibly, the characteristic field strength are rather different. The older measurements based on the Zeeman effect in the quiet Sun, such as those by Grossmann-Doerth et al. (1996); Keller et al. (1994); Sigwarth et al. (1999), have rather low sensitivity and a high noise level and are thus detected mainly network fields. This is probably also true in the case of the observations analyzed in Sánchez Almeida \& Lites (2000), where the profiles selected for analysis are located in the network and its immediate surrounding. By decreasing the threshold limit, Socas-Navarro \& Sánchez Almeida (2002) re-analyzed the same dataset including more inter-network fields. The results on the inter-network fields with the network being intentionally avoided are presented by Domínguez Cerdeña et al. (2003); Khomenko et al. (2003, 2005a); Lin (1995); Lin \& Rimmele (1999); Lites \& Socas-Navarro (2004); Lites (2002); Lites et al. (1996); Martínez González et al. (2006); Sánchez Almeida et al. (2003b); Socas-Navarro et al. (2004).

The fields observed in the inter-network with the Zeeman effect show a strong dependence on granulation. Figure 2 gives an example of such dependence from the internetwork observations in Fe I $1.56 \mu \mathrm{m}$ lines analyzed in Khomenko et al. (2003). The Stokes $V$ splitting gets larger in the dark intergranular lanes. The magnetic field is more intense when the flow of material is downward and is weaker when the flow is upward (Domínguez Cerdeña et al. (2003); Khomenko et al. (2003); Lin \& Rimmele (1999)). Theoretically, the scattering polarization signals should also show a dependence on the granulation structure. Trujillo Bueno et al. (2004) have demonstrated that the degree of the anisotropy of the continuum radiation field at $5000 \AA$ is larger in the upflowing regions of the quiet solar photosphere. The number density of $C_{2}$ molecules is also greater in the upflows above a given height. Thus, the significant linear polarization in $C_{2}$ lines due to the anisotropic pumping should come mainly from these regions. Such theoretical calculations suggest a tool for measuring weak turbulent magnetic fields in the "granular" upflowing zones (Asensio Ramos \& Trujillo Bueno (2005); Trujillo Bueno et al. (2004)). High-resolution scattering polarization observations are needed to confirm whether such a correlation between the scattering polarization amplitude and the granular velocity field is indeed present in the Sun.

\section{ASYMMETRY OF STOKES SPECTRA AS A DIAGNOSTIC TOOL}

Stokes profiles observed in the quiet Sun are asymmetric. The asymmetry of Stokes $V$ reflects gradients of the LOS components of velocity and magnetic field vector (Illing et al. (1975)) and can be used as a diagnostic tool for these parameters.

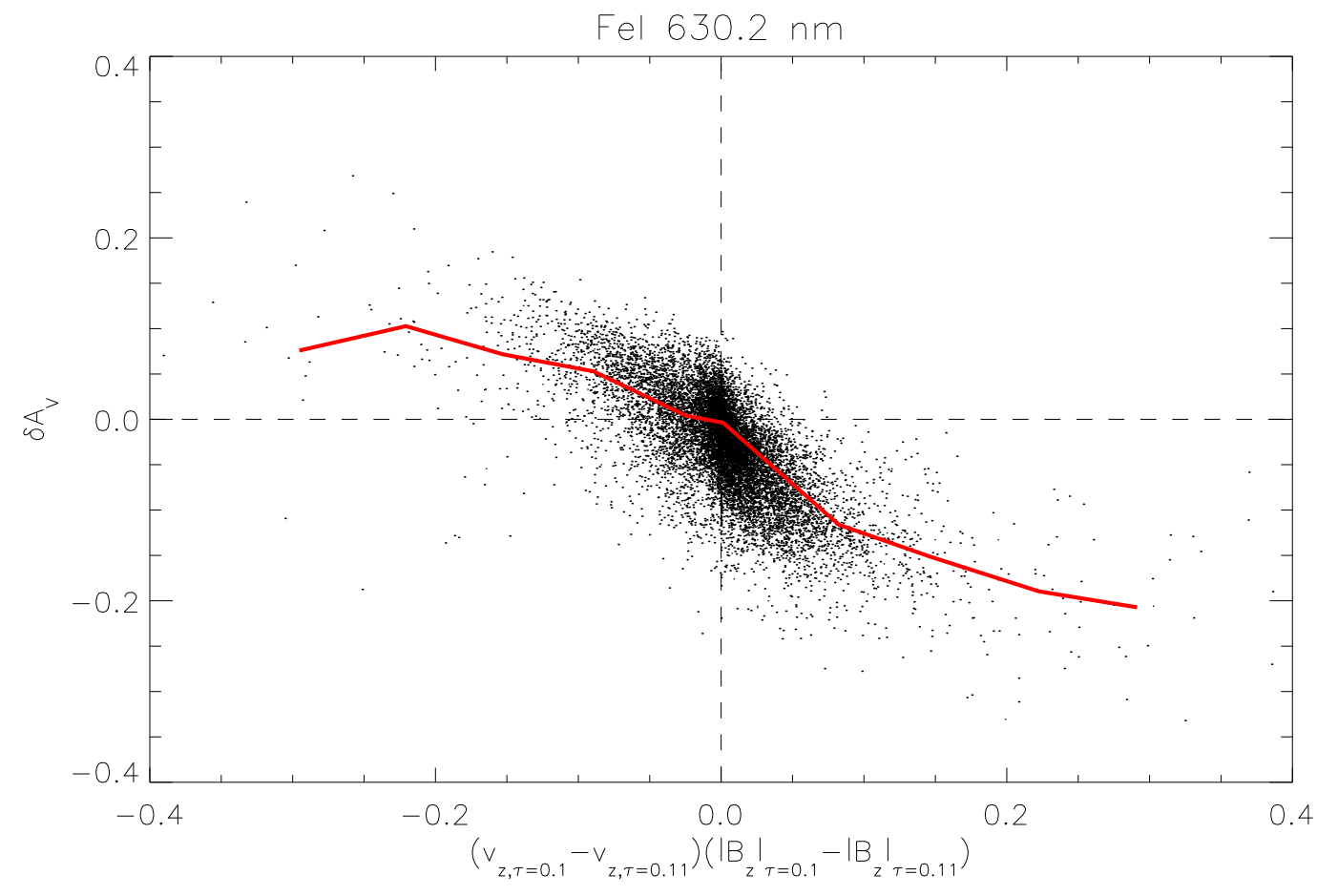

Fig. 3. Scatter plot of the normalized Stokes $V$ area asymmetry versus the product of the gradients of the vertical components of the magnetic field strength and velocity calculated for the Fe I $6302 \AA$ line in the MHD simulations of Vögler et al. (2005). From Shelyag \& et al. (2006). 


\section{E. KHOMENKO, R. KOSTIK}

The asymmetry of Stokes $V$ at the quiet Sun disk center is positive for both network and inter-network fields (defined as $\delta a=\left(a_{b}-a_{r}\right) /\left(a_{b}+a_{r}\right)$, where subscript "b" and "r" denote the blue and red wing, respectively). The amplitude asymmetry usually exceeds area asymmetry (see e.g., Grossmann-Doerth et al. (1996); Khomenko et al. (2003); Sigwarth et al. (1999)). The positive area asymmetry can be explained on the basis of a schematic model of a flux tube with the field lines fanning out with height and a combination of flows inside and outside this feature (Bellot Rubio et al. (1997); Frutiger \& Solanki (1998); Grossmann-Doerth et al. (1988, 1989); Solanki (1989)). If the flow and the magnetic field overlap spatially, the sign of the asymmetry depends on the sign of the velocity and magnetic field gradients at the heights of formation of the line wings (Sánchez Almeida et al. (1989); Solanki \& Pahlke (1988)). The asymmetry is positive if the product of this gradients is negative.

Alternatively, the concept of MISMA was shown to explain any type of asymmetric profile observed in the quiet Sun (Sánchez Almeida et al. (1996)). The MISMA model assumes a set of magnetic components with different polarity and velocity co-existing in the resolution element.

The picture suggested by MHD simulations is significantly more complex, but conceptually similar to the classical flux tube (Shelyag \& et al. (2006)). The strong magnetic field concentrations in intergranular lanes produce profiles with a negative or zero asymmetry inside the feature and a positive asymmetry in the canopy regions (Khomenko et al. (2005b)). Figure 3 shows the scatter plot of the Stokes $V$ area asymmetry of the Fe I $6302 \AA$ line in the MHD simulations of Vögler et al. (2005) as a function of the product of the gradients of vertical components of magnetic field and velocity (Shelyag $\&$ et al. (2006)). It demonstrates that, on average, the asymmetry grows with the increase in the product of the gradients and has a sign that agrees with the expectations from the theoretical predictions based on simple radiative transfer reasonings (Sánchez Almeida et al. (1989); Solanki \& Pahlke (1988)).

Realistic MHD simulations produce, on average, positive area and amplitude asymmetry of Stokes $V$ profiles (Khomenko et al. (2005b)). In the case of the spectra computed with the original numerical resolution, both area and amplitude asymmetry have the same order of magnitude. Profiles in upflowing regions are characterized on average by positive area asymmetries, while profiles in downflowing regions have negative asymmetries, although these are on average weaker. Similar behaviour was first noted in 2D simulations by Sheminova (2003). Recently Socas-Navarro et al. (2004) studied weak polarization signals in Fe I $6302 \AA$ observed in an inter-network region and showed that, on average, over granules Stokes $V$ profiles are characterized by a strong positive asymmetry, while in intergranules the asymmetry is negative and weaker. Thus, the simulations are in qualitative agreement with these observations, despite the difference in the spatial resolution.

The spatial smearing leads to a significant increase of the average amplitude asymmetry, while the area asymmetry does not change (Khomenko et al. (2005b)). The dominance of amplitude asymmetry over area asymmetry is in good agreement with observations. Note that in the idealized turbulent dynamo simulations of Emonet \& Cattaneo (2001) analyzed by Sánchez Almeida et al. (2003a) the average asymmetry is close to zero.

Summarizing all the above, several conclusions may be drawn: 1) the schematic picture of a fluxtube producing positive area asymmetry in the canopy zones is confirmed by the 3D MHD simulations; 2) the area asymmetry is positive in upflows and is negative in downflows; 3) on average, both area and amplitude asymmetries due to the vertical gradients are positive; 4) horizontal spatial smearing makes the observed amplitude asymmetry larger than the area asymmetry.

\section{MAGNETIC FIELD STRENGTH AND FLUX IN INTER-NETWORK}

The issue of characteristic field strength and the true amount flux contained in the inter-network fields remains contradictory.

There are several sources of uncertainties that affect the determination of the inter-network magnetic fields. Firstly, the polarimetric signals are very weak, of the order of $10^{-2}-10^{-3}$ in units of the continuum intensity. Thus, noise and insufficient polarimetric sensitivity do not allow the weakest signals to be reliably detected and analyzed. Secondly, the spatial structure of the magnetic fields remains unresolved even in the best-resolution observations. As a consequence, the measurements of the Zeeman effect can be affected by polarity cancellations, leading to non-detection of a (possibly) significant part of the flux. Finally, the weak profiles in inter-network are strongly asymmetric and have irregular shapes. The interpretation of such kinds of spectra in terms of simplified models often lead to confusion and contradictions between the different measurements.

The unsigned longitudinal magnetic flux in the internetwork measured with the Zeeman effect remains typically within 6-9 G at 1 arcsec resolution (see, for example, Khomenko et al. (2003); Lites \& Socas-Navarro (2004); Lites (2002); Sánchez Almeida et al. (2003b)). These, however, are not true values, since a part of flux can be hidden due to unresolved polarities. One possible way of overcoming this disadvantage of Zeeman measurements is to compare MHD simulations with observations. This comparison is based on a unique relation between the flux in a region and the amplitudes of Stokes $V$ measured there. The direct comparison of the amplitudes of Stokes $V$ in the simultaneous observations in the IR $1.5648 \mu \mathrm{m}$ and visible $6302 \AA$ Fe I lines with the MHD simulations allowed Khomenko et al. (2005a) to conclude that the "true" value of mean magnetic field strength in inter-network is of $20 \mathrm{G}$. This corresponds to an average longitudinal flux of $11-15 \mathrm{G}$ at $\log \tau_{5}$ from -1 to 0 .

The occupation fraction of the fields measured with the Zeeman effect is usually a few per cent. It becomes 
clear now that the rest of the volume is not fully fieldfree, but can be occupied by turbulent fields. The average strength of such fields can be measured with the help of Hanle-sensitive spectropolarimetric data. The most rigorous modeling of the Hanle effect performed until now is that of Trujillo Bueno et al. (2004). A comparison of the 3D multilevel radiative transfer in the Sr I $4607 \AA$ line, performed in a 3D HD model atmosphere of Asplund et al. (2000), with observations allowed the authors to conclude that the average field strength in the quiet Sun can be of the order of $100 \mathrm{G}$.

Thus, there is a contradiction between the Hanle and the Zeeman measurements of the magnetic flux. However, it should be kept in mind that the results of both Khomenko et al. (2005a) and Trujillo Bueno et al. (2004) are model-dependent and probably suffer from the lack of turbulent component of the magnetic and velocity fields in the numerical simulations due to the insufficiently high magnetic Reynolds number (Vögler et al. (2005)).

At the same time, there is no agreement between the magnetic field strength distribution measured in internetwork with the help of the different spectral lines. The infrared Fe I lines at $1.56 \mu \mathrm{m}$ reveal mostly weak fields with an exponential distribution (Domínguez Cerdeña et al. (2006); Khomenko et al. (2003); Lin (1995); Lin \& Rimmele (1999); Martínez González et al. (2006)). While the visible Fe I 6301 and $6302 \AA$ lines suggest that the characteristic field strength is kG (Domínguez Cerdeña et al. (2003, 2006); Lites (2002); Sánchez Almeida et al. (2003b); Socas-Navarro \& Sánchez Almeida (2002)).
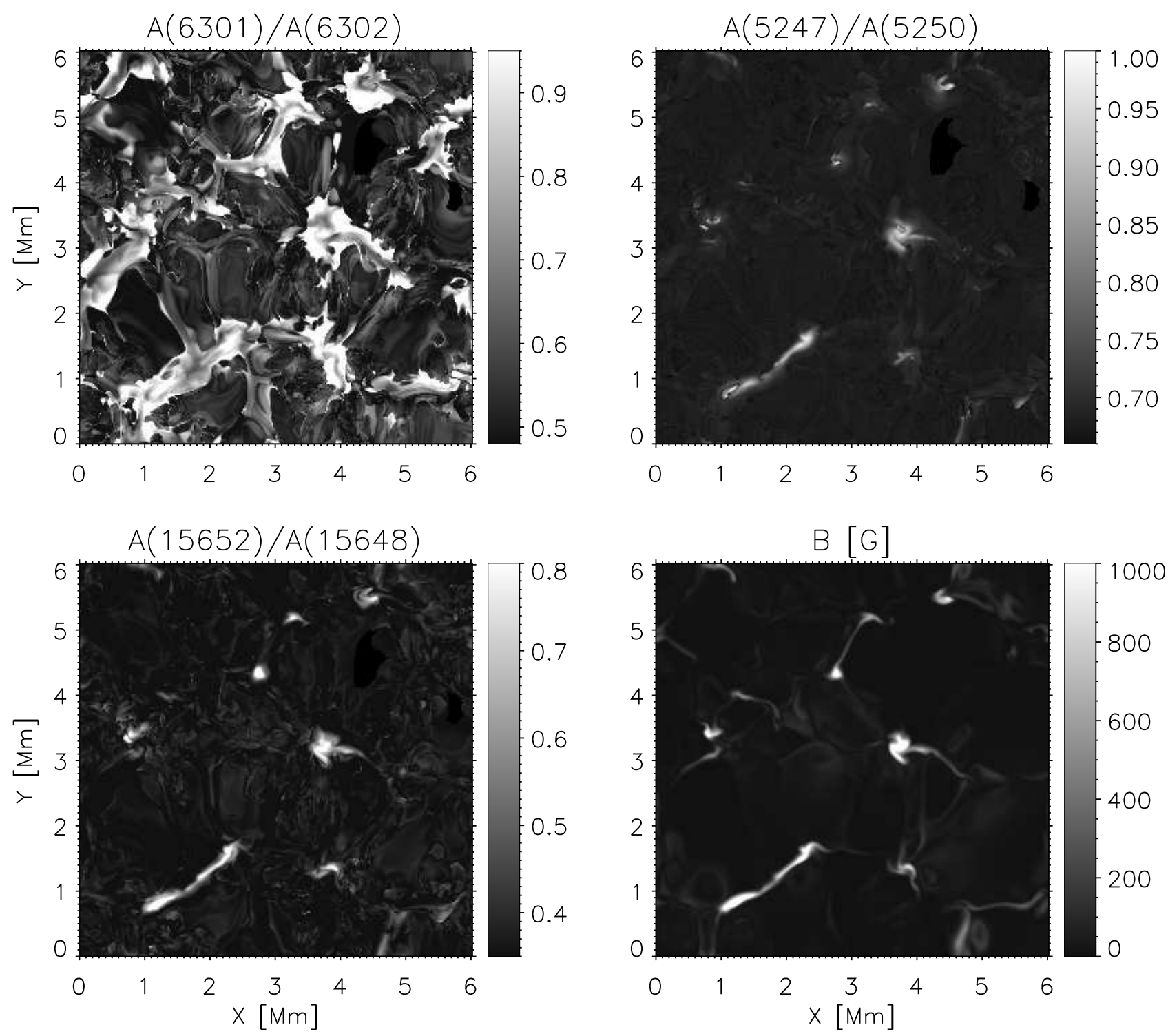

Fig. 4. Ratio of the Stokes $V$ amplitudes of the Fe I 6301 and $6302 \AA$ lines (top left), Fe I 5247 and $5250 \AA$ lines (top right) and Fe 15652 and $15648 \AA$ lines (bottom left). Bottom right panel: map of the magnetic field strength at $\log \tau_{5}=-1$ in the $30 \mathrm{G}$ flux snapshot from the simulations of Vögler et al. (2005). Bright pixels are the pixels where the kG field strength would be recovered if we measure the original field distribution with the corresponding pair of lines. 


\section{E. KHOMENKO, R. KOSTIK}

The reason for this difference could be, on the one hand, the noise present in the observational data. Bellot Rubio \& Collados (2003) have demonstrated that noise can affect the results of the Stokes inversion in a way that the maximum of the PDF obtained from the visible lines may be shifted to peak in the $\mathrm{kG}$ range, while the IR lines seem to recover the original exponential PDF reasonably well.

On the other hand, the interpretation of the data could be affected by the different Zeeman sensitivity in the two spectral regions. A numerical experiment performed by Socas-Navarro \& Sánchez Almeida (2003) suggests that if there are at least two - weak and strong - components of the magnetic field co-existing in the resolution element, then the IR lines tend to detect the weak component while the visible lines are selective to the strong component. This happens because the visible lines form in weak-field conditions for the fields as high as 1-1.5 $\mathrm{kG}$, while the infrared lines already enter in a strong-field regime for the fields above $500-800 \mathrm{G}$. Thus, a profile resulting from a combination of the two components, one weak (with a larger filling factor) and one strong (with a smaller filling factor), in the infrared would have the same amplitude as a weak-field profile accompanied by additional lobes. These lobes would, likely, remain below the noise level in observations due to their small amplitude. A fit to this profile would reveal only the weak component. In the visible, such a composite profile would change its amplitude instead of its shape. Thus, the relative amplitude of the Fe I 6301 and $6302 \AA$ lines would be modified in the direction appropriate for the strong fields. A fit to this line pair would reveal some weighted average between the weak and strong field components. For the details see Socas-Navarro \& Sánchez Almeida (2003).

Eventually, the conclusion on whether or not the characteristic field strength in inter-network is $\mathrm{kG}$ depends on the reliability of the diagnostic techniques applied to the observations. The results of the line ratio or magnetogram calibration and Milne-Eddington inversions depend on the assumption that the gradients of parameters such as temperature, velocity and magnetic fields are absent. However, this approach is only valid if the spectral lines used for the inversion have exactly the same sensitivity to all atmospheric parameters, except for the magnetic field, and form at the same height. Figure 4 gives a test calculation of the Stokes $V$ amplitude ratio performed in a snapshot of the MHD simulations of Vögler et al. (2005) for the different pairs of lines. For simplicity, the profiles are taken at their original numerical resolution of $20 \mathrm{~km}$ and no noise is added. The values of the ratio of Stokes $V$ amplitudes of the pair of lines depend (among other parameters) on the field strength and atomic parameters of the spectral lines used. In the simplest case of constant magnetic field, this ratio changes within $0.5-0.95$ for the Fe I 6301, $6302 \AA$ lines, 0.67-1.0 for the Fe I $5247,5250 \AA$ lines and $0.37-0.8$ for the IR lines, where the first value corresponds to the case of a weak field and the second value corresponds to the case of a strong field. Note, that these values are calculated assuming both $V_{\text {mic }}$ and $V_{\text {mac }}$ equal to zero. The line ratios presented in Fig. 4 are scaled in such a way that light colors would correspond to a kG field strength. The original "true" snapshot is shown in the bottom right panel. It follows that the locations with strong fields in the original snapshot correspond rather well to locations with maximum line ratio for the Fe I 5247, 5250 and 1568, 15652 line pairs. This is not the case for the Fe I 6301, $6302 \AA$ lines. There, the line ratio is greatest not where the field is the largest, but rather in the canopy regions surrounding the magnetic field concentrations and in the transition regions between granules and intergranules. The reason for such a behavior is a large difference in the heights of the formation of the Fe I 6301, $6302 \AA$ lines: on average, about $100 \mathrm{~km}$ (Shchukina \& Trujillo Bueno (2001)). The vertical gradients of the magnetic field and velocity and the horizontal fluctuations of the temperature around the snapshot produce a distortion of the line ratio of these lines in the way that appears in Figure 4. This does not happen for the Fe I 5247, 5250 and 1568, 15652 $\AA$ lines, whose heights of formation are close enough and for which the assumption about the absence of gradients is not crucial. Thus, the results of magnetic field measurements based on the line ratios or magnetograms of the Fe I 6301, 6302 lines should be viewed with caution. The latter probably applies to Milne-Eddington inversions as well. The way of treating the gradients in the inversion of these lines affects the conclusions concerning the magnetic field distribution in the quiet Sun in a significant way.

\section{CONCLUSIONS}

The resolution of modern ground-based and space observations has reached a detection of small scale variations of the magnetic field on the Sun of the order of 100-200 km. However, even such a high resolution does not seem to be high enough to completely resolve the spatial structuring of the magnetic field. The MHD simulations show extremely complex structures on scales close to their numerical resolution of a few tens of $\mathrm{km}$. But the presence of this turbulent component in the simulations is limited by problems of numerical stability and an insufficiently high Reynolds number. Thus, the present simulations probably do not resolve the topology of the field as well.

The simplified diagnostic methods applied in the analysis of such complex fields at high resolution often fail in giving reliable results. The noise level and the insufficient resolution make the weak quiet Sun signals compatible with different physically reasonable scenarios. The results of the inversion depend on the treatment of the asymmetries (as, for example, in the case of the Fe I 6301, $6302 \AA$ lines). Thus, a careful selection of lines should be made for the investigation of the weak inter-network fields. Several types of diagnostics, based on the Zeeman and Hanle effects in atomic and molecular lines, should be combined together to constrain the results.

Future work is needed to answer open questions such as the following: 
- What is the characteristic field strength and shape of the magnetic field distribution in the quiet Sun?

- How large is the magnetic energy contained in the quiet Sun and how important is it for the energy balance of the upper solar atmosphere?

- What process generates the quiet Sun magnetic fields that are present independently of the solar cycle?

Acknowledgements We are grateful to our colleagues of the IAC solar group for their help and interesting discussions. This research was partly funded by the Spanish Ministerio de Educación y Ciencia through project AYA2004-05792.
[1] A. Asensio Ramos, J. Trujillo Bueno, Astrophys. J. 635, L109 (2005).

[2] M. Asplund, A. Nordlund, R. Trampedach, C. Allende Prieto, R. F. Stein, Astron. Astrophys., 359, 729 (2000).

[3] L. R. Bellot Rubio, B. Ruiz Cobo, M. Collados, Astrophys. J. 478, L45 (1997).

[4] L. R. Bellot Rubio, M. Collados, Astron. Astrophys. 406, 357 (2003).

[5] T. E. Berger, L. H. M. Rouppe van der Voort, Löfdahl et al., Astron. Astrophys. 428, 613 (2004).

[6] V. Bommier, M. Derouich, E. Landi Degl'Innochenti, G. Molodij, S. Sahal-Bréchot, Astron. Astrophys. 432, 295 (2005).

[7] M. Carlsson, R. F. Stein, A. Nordlund, G. B. Scharmer, Astrophys. J. 610, L137 (2004).

[8] I. Domínguez Cerdeña, J. Sánchez Almeida, F. Kneer, Astron. Astrophys. 407, 741 (2003).

[9] I. Domínguez Cerdeña, J. Sánchez Almeida, F. Kneer, Astrophys. J. 636, 496 (2006).

[10] T. Emonet, F. Cattaneo, Astrophys. J. 560, L197 (2001).

[11] M. Faurobert, J. Arnaud, J. Vigneau, H. Frisch, Astron. Astrophys. 378, 627 (2001).

[12] M. Faurobert, N. Feautrier, F. Machefert, K. Petrovay, A. Spielfiedel, Astron. Astrophys. 298, 289 (1995).

[13] C. Frutiger, S. K. Solanki, Astron. Astrophys. 336, L65 (1998).

[14] C. Frutiger, S. K. Solanki, M. Fligge, J. H. M. J. Bruls, in Solar Polarization, Vol. 243, edited by K. N. Nagendra, J. O. Stenflo (Kluwer, Dordrecht, 1999), p. 281.

[15] C. C. Frutiger, S. K. Solanki, M. Fligge, J. H. M. J. Bruls, Astron. Astrophys. 58, 1109 (2000).

[16] A. S. Gadun, S. K. Solanki, V. A. Sheminova, S. R. O. Ploner, Solar Phys. 203, 1 (2001).

[17] U. Grossmann-Doerth, C. U. Keller, M. Schüssler, Astron. Astrophys. 315, 610 (1996).

[18] U. Grossmann-Doerth, M. Schüssler, S. K. Solanki, Solar Phys. 206, L37 (1988).

[19] U. Grossmann-Doerth, M. Schüssler, S. K. Solanki, Astron. Astrophys. 221, 338 (1989)

[20] U. Grossmann-Doerth, M. Schüssler, O. Steiner, Astron. Astrophys. 337, 928 (1998).
[21] R. M. E. Illing, D. A. Landman, D. L. Mickey, Astron. Astrophys. 41, 183 (1975).

[22] C. Keller, M. Schüssler, A. Vögler, V. Zakharov, Astrophys. J. 607, L59 (2004).

[23] C. U. Keller, F.-L. Deubner, U. Egger, B. Fleck, H. P. Povel, Astron. Astrophys. 286, 626 (1994).

[24] E. V. Khomenko, M. Collados, S. K. Solanki, A. Lagg, J. Trujillo Bueno, Astron. Astrophys. 408, 1115 (2003).

[25] E. V. Khomenko, M. J. Martínez Gonzalez, M. Collados et al. Astron. Astrophys. 436, L27 (2005).

[26] E. V. Khomenko, S. Shelyag, S. K. Solanki, A. Vögler, Astron. Astrophys. 442, 1059 (2005).

[27] E. Landi Degl'Innocenti, Solar Observations: Techniques and Interpretation, edited by F. Sánchez, M. Collados, M. Vázquez (Cambridge University press, 1992), p. 73

[28] E. Landi Degl'Innocenti, M. Landolfi, Polarization in Spectral Lines (Kluwer Academic Publishers, Dordrecht, 2004).

[29] H. Lin, Astrophys. J. 446, 421 (1995).

[30] H. Lin, T. Rimmele, Astrophys. J. 514, 448 (1999).

[31] B. Lites, H. Socas-Navarro, Astrophys. J. 613, 600 (2004).

[32] B. W. Lites, Astrophys. J. 573, 431 (2002).

[33] B. W. Lites, K. D. Leka, A. Skumanich, V. Martínez Pillet, T. Shimizu, Astrophys. J. 460, 1019 (1996).

[34] A. López Ariste, S. Tomczyk, R. Casini, Astrophys. J. 580, 519 (2002).

[35] A. López Ariste, S. Tomczyk, R. Casini, Solar Polarization 4, edited by R. Casini, B. W. Lites, ASP Conf. Series 358, 54 (2006).

[36] R. Manso Sainz, E. Landi Degl'Innochenti, J. Trujillo Bueno, Astron. Astrophys. 447, 1125 (2006).

[37] M. J. Martínez González, M. Collados, B. Ruiz Cobo, Astron. Astrophys. 456, 1159 (2006).

[38] D. N. Rachkovsky, Izv. Krymsk. Astrofiz. Obs. 37, 56 (1967).

[39] D. E. Rees, A. López Ariste, J. Thatcher, M. Semel, Astron. Astrophys. 355, 759 (2000).

[40] B. Ruiz Cobo, J. C. del Toro Iniesta, Astrophys. J. 398, 375 (1992)

[41] B. Ruiz Cobo, J. C. del Toro Iniesta, Astron. Astrophys. 283, 129 (1994) 


\section{E. KHOMENKO, R. KOSTIK}

[42] J. Sánchez Almeida, M. Collados, J. C. del Toro Iniesta, Astron. Astrophys. 222, 311 (1989).

[43] J. Sánchez Almeida, E. Landi degl'Innocenti, V. Martinez Pillet, B. W. Lites, Astrophys. J. 466, 537 (1996).

[44] J. Sánchez Almeida, Astrophys. J. 491, 993 (1997).

[45] J. Sánchez Almeida, B. W. Lites, Astrophys. J. 532, 1215 (2000).

[46] J. Sánchez Almeida, T. Emonet, F. Cattaneo, Astrophys. J. 585, 536 (2003).

[47] J. Sánchez Almeida, I. Domínguez Cerdeña, F. Kneer, Astrophys. J. 597, L177 (2003).

[48] W. Schaffenberger, S. Wedemeyer-Böhm, O. Steiner, B. Freytag, in Proceedings of the International Scientific Conference on Chromospheric and Coronal magnetic fields (ESA SP-596), Katlenburg-Lindau, 2005, edited by D. E. Innes, A. Lagg, S. K. Solanki (CDROM, Katlenburg-Lindau, 2006), p. 65.1.

[49] M. Schüssler, Advanced solar polarimetry: Theory, observation and instrumentetion, edited by M. Sigwarth, ASP Conf. Series 236, 343 (2001).

[50] M. Schüssler, Solar Polarization, edited by in J. Trujillo Bueno, J. Sánchez Almeida, ASP Conf. Series 307, 601 (2003).

[51] M. Schüssler, S. Shelyag, S. Berdyugina, A. Vöegler, S. K. Solanki, Astrophys. J. 597, L173 (2003).

[52] N. G. Shchukina, J. Trujillo Bueno, Astrophys. J. 550, 970 (2001).

[53] S. Shelyag, M. Schuessler, S. K. Solanki, A. Voegler, Astronom. Astrophys., 2007 (in press).

[54] V. A. Sheminova, Kinem. Fiz. Nebes. Til 19, є 2, 107 (2003).

[55] V. A. Sheminova, Kinem. Fiz. Nebes. Til 20, e 1, 3 (2004).

[56] M. Sigwarth, K. S. Balasubramaniam, M. Knölker, W. Schmidt, Astron. Astrophys. 349, 941 (1999).

[57] A. Skumanich, B. W. Lites, Astrophys. J. 322, 473 (1987).

[58] H. Socas-Navarro, B. Ruiz Cobo, J. Trujillo Bueno, Astrophys. J. 507, 470 (1998).

[59] H. Socas-Navarro, J. Trujillo Bueno, B. Ruiz Cobo, Astrophys. J. 530, 977 (2000).

[60] H. Socas-Navarro, in Advanced Solar Polarimetry: Theory, Observation, and Instrumentation, edited by M. Sigwarth, ASP Conf. Series 236, 487 (2001).

[61] H. Socas-Navarro, A. López Ariste, B. W. Lites, Astrophys. J. 553, 949 (2001).

[62] H. Socas-Navarro, J. Sánchez Almeida, Astrophys. J. 565, 1323 (2002).

[63] H. Socas-Navarro, J. Sánchez Almeida, Astrophys. J. 593, 581 (2003).
[64] H. Socas-Navarro, V. Martínez Pillet, B. Lites, Astrophys. J. 611, 1139 (2004).

[65] H. Socas-Navarro, Astrophys. J. 633, L57 (2005).

[66] H. Socas-Navarro, Astrophys. J. 621, 545 (2005).

[67] S. K. Solanki, Astron. Astrophys. 224, 225 (1989).

[68] S. K. Solanki, Space Science Reviews 63, 1 (1993).

[69] S. K. Solanki, K. D. Pahlke, Astron. Astrophys. 201, 143 (1988).

[70] R. F. Stein, A. Nordlund, in Modelling of Stellar Atmospheres, edited by N. E. Piskunov, N. W. Weiss, D. E. Gray, ASP Conf. Series 210169 (2003).

[71] J. O. Stenflo, Solar Phys. 32, 41 (1973).

[72] J. C. del Toro Iniesta, B. Ruiz Cobo, Solar Phys. 164, 169 (1996).

[73] J. Trujillo Bueno, in Advanced Solar Polarimetry: Theory, Observation, and Instrumentation, edited by M. Sigwarth, ASP Conf. Series 236, 161 (2001).

[74] J. Trujillo Bueno, in Stellar Atmosphere Modeling, edited by I. Hubeny, D. Mihalas, K. Werner, ASP Conf. Series 288, 551 (2003).

[75] J. Trujillo Bueno, in Solar Polarization, edited by Sánchez Almeida, J. Trujillo Bueno ASP Conf. Series 307, 407, (2003).

[76] J. Trujillo Bueno, R. Manso Sainz, Nuovo Cimento, 25 C, No. 5-6, 783 (2002).

[77] J. Trujillo Bueno, E. Landi Degl'Innocenti, M. Collados, L. Merenda, R. Manso Sainz, Nature 415, 403 (2002).

[78] J. Trujillo Bueno, N. G. Shchukina, A. Asensio Ramos, Nature 430, 326 (2004).

[79] J. Trujillo Bueno, L. Merenda, R. Centeno, M. Collados, E. Landi Degl'Innocenti, Astrophys. J. 619, L191 (2005).

[80] W. Unno, Publ. Astron. Soc. Jpn 8, 108 (1956).

[81] A. Vögler, S. Shelyag, M. Schüssler, F. Cattaneo, T. Emonet, T. Linde, Astron. Astrophys. 429, 335 (2005).

[82] S. Wedemeyer-Böhm, W. Schaffenberger, O. Steiner, M. Steffen, B. Freytag, I. Kamp, in Proceedings of the International Scientific Conference on Chromospheric and Coronal magnetic fields (ESA SP596), Katlenburg-Lindau, 2005, edited by D. E. Innes, A. Lagg, S. K. Solanki (CDROM, Katlenburg-Lindau, 2006), p. 65.1

[83] C. Westendorp Plaza, J. C. del Toro Iniesta, B. Ruiz Cobo, V. Martínez Pillet, B. W. Lites, A. Skumanich, Astrophys. J. 494, 453 (1998). 


\title{
ДОСЛІДЖЕННЯ МАГНЕТИЗМУ СПОКІЙНОГО СОНЦЯ: ТЕХНІКА ВИМІРЮВАНЬ ТА РЕЗУЛЬТАТИ
}

\author{
О. Хоменко ${ }^{1,2}$, Р. Костик ${ }^{2}$ \\ ${ }^{1}$ Інститут Астрофізики на Канарах, Ла Лаљ'уна, Тенерифе, Е-38200, Іспанія \\ ${ }^{2}$ Головна астрономічна обсерваторія НАН Украӥни, вул. Заболотного, 27, Київ, 03680, Україна
}

Значна частина наших знань про магнетизм поверхні Сонця отримана з аналізу спектра поляризованого світла. Параметри Стокса містять докладну інформацію про структуру й динаміку замагнеченої фотосферної плазми, а також про її взаємодію з конвекцією, т. зв. магнетоконвекція. При інтерпретацї спостережних спектрів, які одержані з високою роздільною здатністю, потрібно застосовувати складну теорію радіятивного переносу поляризованого світла у тривимірних моделях. 3 іншого боку, щоб реалістично описати поведінку плазми в замагнеченій атмосфері Сонця, тривимірне моделювання вимагає детального врахування складних фізичних процесів. У цій праці ми робимо наголос на діягностику магнетних полів у спокійних фотосферних ділянках за межами сонячних плям та активних ділянок. Донедавна вважали, що магнетне поле суттєво не впливає на динаміку плазми в цих ділянках. Однак саме в спокійних ділянках накопичена більша частина магнетної енергіï. Тому питання про магнетизм спокійного Сонця і надалі відкрите й широко дебатується в літературі. 